\title{
3 Zielstellung
}

Der BDH Bundesverband Rehabilitation e.V. hat das Promotiosstipendium mit dem Ziel ausgeschrieben, die therapeutische Pflege in der neurologischen Frührehabilitation zu untersuchen. Dabei waren vor allem die Fragen leitend: „Welche Handlungen führen Pflegende in der neurologischen Frührehabilitation durch?" Wie führen Pflegende diese Handlungen durch?“ Was ist das Therapeutische an diesen Handlungen?“ In gemeinsamen Besprechungen wurde vereinbart, dass sowohl die Phase $\mathrm{B}$ als auch die Phase $\mathrm{C}$ in die Untersuchung eingeschlossen werden. Weiterhin wurde auf Basis der vorangestellten Fragestellungen entschieden, eine materiale Pflegetheorie (Erklärung unter Punkt 4.6.1 Materiale oder formale Theorie) zur therapeutischen Pflege in der neurologischen (Früh) Rehabilitation (Phasen B und C) zu entwickeln, welche diese Fragestellungen beantworten wird. 